\title{
¿Qué Podemos Aprender de los Sistemas Educativos de Chile, España y Finlandia en el Marco de la Declaración de Salamanca?
}

\section{¿What Can We Learn from Educational Systems of Chile, Spain and Finland in the Framework of Salamanca Statement?}

\author{
Ricardo Rosas ${ }^{1 *}$ \\ James Staig ${ }^{1}$ \\ Guillermo Lazcano ${ }^{1}$ \\ Rosario Palacios ${ }^{1}$ \\ Victoria Espinoza ${ }^{1}$ \\ Mikko Aro ${ }^{2}$ \\ Candelaria Imbernón ${ }^{3}$ \\ ${ }^{1}$ Pontificia Universidad Católica de Chile, Chile \\ 2 Jyvaskyla University, Finlandia \\ ${ }^{3}$ Centro Universitario La Salle, España
}

\begin{abstract}
El presente artículo compara los sistemas de Educación Especial de Chile, España y Finlandia, desde la perspectiva de la Declaración de Salamanca para las Necesidades Educativas Especiales (NEE). Tanto el contexto general de sus sistemas educativos como su orientación epistemológica respecto de la Educación Especial, los marcos regulatorios de cada sistema, los procedimientos de detección y derivación de niños con NEE, y la estructura de los servicios de atención a la diversidad, dialogan de manera particular con los compromisos adquiridos en 1994. Se muestran las principales diferencias entre los sistemas, destacando la gran variabilidad respecto de la definición de NEE y sus estrategias de inclusión de estudiantes de NEE al sistema escolar. Los resultados de esta investigación muestran diferencias importantes en el logro de los objetivos planteados por la declaración, en los tres países estudiados, estando Chile aún muy lejos de contar con un sistema de atención a la diversidad verdaderamente inclusivo. Los desafíos pendientes para nuestro país son que no cuenta con un marco regulatorio integrado para la atención a la diversidad; cuenta con provisión de recursos humanos y tecnológicos muy limitados para estudiantes con NEE, y tiene una insuficiente cobertura de las NEE en la formación inicial docente.
\end{abstract}

Descriptores: Sistema educativo; Educación especial; Educación comparada; Educación integradora; Declaración de Salamanca.

This paper compares the Special Education Systems form Chile, Spain and Finland, from the perspective of the UNESCO Salamanca Statement on Special Education Needs (SEN). The following issues create a dialogue about the agreement reached in 1994: the general context of the educational systems of each country, their epistemological orientation regarding Special Education, the regulatory framework of each system, the procedures to detect and refer children with SEN, and the structure of the diversity services. This paper will cover the main differences between the systems, highlighting the great diversity in the definition of Special Educational Needs and the strategies for the inclusion of SEN students into the regular school system. The results of this research show mayor differences on the fulfillment of objectives established by the statement on all three countries covered

*Contacto: rrosas@uc.cl

ISSN: 0718-7378

www.rinace.net/rlei/
Recibido: $\quad$ 13/09/2019

$1^{a}$ Evaluación: 30/09/2019

Aceptado: 11/10/2019 
by the study, Chile being well behind on having a diversity system that can be considered truly inclusive. The challenges that remain for our country are that we do not have an integrated regulatory framework for diversity work; we have very limited technological and human resources for students with SEN, and there is not enough coverage of SEN in the early stages of teacher training.

Keywords: Educational system; Special education; Comparative education; Inclusive education, Salamanca statement.

\section{Presentación}

A 25 años de la Declaración de Salamanca, vivimos momentos de cambios importantes en las políticas de educación inclusiva en nuestro país. Como decíamos en una publicación reciente (Rosas, Staig y Lazcano, 2017), nuestro sistema educacional avanza hacia una mayor inclusividad de estudiantes con NEE, aunque aún con serias falencias. La Declaración de Salamanca proclama el derecho a la educación ${ }^{1}$, reconoce diferentes características, intereses, capacidades y necesidades de aprendizaje en $\operatorname{los}_{n i n ̃ o s}{ }^{2}$, y en esa línea establece la necesidad de que existan formas de enseñanza que tomen en cuenta tales diferencias ${ }^{3}$. A la vez, enfatiza los beneficios de la educación de los niños con NEE en escuelas ordinarias y establece el derecho de ellos al acceso a este tipo de establecimientos4. En este contexto, vemos que el sistema chileno muestra importantes desafíos para adherir de manera más integral a la Declaración, siendo las más importantes a nuestro juicio, a) la necesidad de un marco regulatorio integrado que aúne todas las disposiciones, leyes y reglamentos que definen aspectos tales como el diagnóstico de NEE, las subvenciones especiales, el sistema de Escuelas de Educación Especial, etc. Esta falencia contraviene el llamado de Salamanca a los gobiernos a dar prioridad política (y presupuestaria) al mejoramiento de sistemas inclusivos, adoptando como ley o política pública el principio de educación integrada (acoger a todos los niños aceptando y celebrando la diferencia), creando mecanismos de planificación, supervisión y evaluación; b) una provisión limitada de recursos en sistemas de mediación, humana y tecnológica, que permitan una verdadera inclusión de alumnos en situación de discapacidad (lectores de pantalla, intérpretes de lengua de señas, inversión en accesibilidad). Esto, además de contravenir la prioridad presupuestaria que mandata la declaración de Salamanca, contraviene las indicaciones del Marco de Acción referentes a que los niños con NEE deben recibir todo el apoyo adicional necesario para su aprendizaje (tanto ayudas en aula como profesionales externos); oportunidad de relacionarse con adultos con discapacidad que puedan mediar el aprendizaje de niños y niñas; y c) una insuficiente consideración del tratamiento de las NEE en la formación inicial docente de los profesores regulares que deberán afrontar las demandas de un aula inclusiva. Esta carencia contraviene el llamado de Salamanca a la

\footnotetext{
1 "todos los niños de ambos sexos tienen un derecho fundamental a la educación y debe dárseles la oportunidad de alcanzar y mantener un nivel aceptable de conocimientos”. (Declaración de Salamanca viii; UNESCO, 1994)

2 "cada niño tiene características, intereses, capacidades y necesidades de aprendizaje que le son propios," (Declaración de Salamanca viii; UNESCO, 1994)

s "los sistemas educativos deben ser diseñados y los programas aplicados de modo que tengan en cuenta toda la gama de esas diferentes características y necesidades". (Declaración de Salamanca viii; UNESCO, 1994)

4. "las personas con necesidades educativas especiales deben tener acceso a las escuelas ordinarias, que deberán integrarlos en una pedagogía centrada en el niño, capaz de satisfacer esas necesidades, las escuelas ordinarias con esta orientación integradora representan el medio más eficaz para combatir las actitudes discriminatorias, crear comunidades de acogida, construir una sociedad integradora y lograr la educación para todos; además, proporcionan una educación efectiva a la mayoría de los niños y mejoran la eficiencia y, en definitiva, la relación costo-eficacia de todo el sistema educativo." (Declaración de Salamanca viii-ix; UNESCO, 1994)
} 
generación de programas orientados hacia las NEE para la formación de profesores y las indicaciones del Marco de Acción sobre la responsabilidad del equipo docente en su conjunto de la enseñanza de niños con NEE y la capacitación pedagógica especializada en NEE.

El objetivo del presente artículo es comparar el sistema educacional inclusivo chileno con el sistema educacional de España y Finlandia, dos países desarrollados que han adherido a la Declaración de Salamanca de maneras distintas. Ambos, han traducido, por así decirlo, los principios establecidos en Salamanca, de manera situada y flexible, para lograr la inclusión de estudiantes con NEE en sus contextos particulares. De ellos, podemos adoptar algunas disposiciones ya que, aunque es un lugar común saber y decir que no es posible copiar modelos extranjeros, el propósito de esta comparación, con estos dos países en particular, obedece a buenas razones. El sistema español ha sido fuente frecuente de inspiración para las reformas educativas en nuestro país desde el retorno a la democracia, y es un sistema que por razones culturales e idiomáticas podría ser un buen modelo a seguir. El sistema finlandés, por otra parte, es tomado como ejemplo a nivel mundial, por sus buenos resultados en educación en general, y por su innovador enfoque en Educación Especial, en particular. Además, ambos países cuentan con una vasta experiencia en modelos de integración e inclusión educativas.

Hemos organizado el artículo de la siguiente manera: partiremos mostrando los tres sistemas en base a una estructura común, partiendo por el contex to general de los sistemas educativos, con énfasis en su orientación epistemológica, particularmente en lo referido a Educación Especial. Este punto nos parece importante dado que el reconocimiento de la diversidad en los estudiantes que realiza Salamanca implica un entendimiento de lo que son las necesidades educativas especiales y de la forma en cómo identificarlas y atenderlas. Consecuentemente, continuaremos describiendo en general los marcos regulatorios de cada sistema, los procedimientos de detección y derivación de niños con NEE, y la estructura de los servicios de atención a la diversidad. Luego, nos centraremos en la inclusividad de los sistemas, tomando en cuenta la fuerza que pone la Declaración en el derecho de los niños a acceder a las escuelas ordinarias y a los beneficios que podrían desprenderse de ello. Finalmente, concluiremos con sugerencias que podrían hacer del sistema chileno un sistema de educación verdaderamente inclusivo.

\section{Una mirada sinóptica a los tres modelos}

\subsection{Modelo chileno}

El sistema de educación escolar chileno tiene como primera base legal la Ley $\mathrm{N}^{\circ} 20.370$ General de Educación (LGE) (2009), en la cual se describe el sistema bajo los 4 parámetros de "equidad y calidad de servicio" (Art. 3). Esta ley, que actúa como la columna vertebral sobre la que se construyen los esfuerzos educacionales escolares en Chile, establece desde el principio que la educación debe de enmarcarse en un "respeto y valoración de los derechos humanos" (Art. 4), valorando así los acuerdos internacionales que ha suscrito nuestro país, que buscan la igualdad de condiciones para todas las personas.

En la ley, se plantean los principios que inspiran al sistema educacional de Chile (Art. 3), de los cuales destacamos (se conserva numeración original): c) Equidad del sistema educativo, e) Diversidad, j) Integración. Este último punto marca la filosofía bajo la cual se entenderá la participación de personas con NEE en la educación nacional. La letra j del 
artículo 3 de la Ley General de Educación dice textualmente "El sistema propiciará la incorporación de alumnos de diversas condiciones sociales, étnicas, religiosas, económicas y culturales" (Ley $\mathrm{N}^{\circ}$ 20.370, 2009). Integración e Inclusión se mueven como dos conceptos intercambiables en la LGE, ya que hacia el final de su Art. 4 se menciona como deber del Estado "velar por [...] la inclusión educativa”, mencionando nuevamente las condiciones presentadas en la cita anterior y un general "entre otras".

A partir de la LGE se establecen modalidades educativas que en el caso de las NEE se derivan en Educación Especial (EE) o Proyectos de Integración Escolar (PIE) (Ley $\mathrm{N}^{\circ} 20.370$, 2009, Art. 22 y 23). Es así como se definen a nivel orgánico del sistema las posibles respuestas a estas NEE de personas en situación de discapacidad. Junto al marco general que se establece en la LGE existen más de una veintena de decretos y otras cinco leyes que abordan temáticas sobre educación y discapacidad, ya sea en la EE o en los PIE, siendo una de las más importantes la Ley $\mathrm{N}^{\circ} 20.845$ de Inclusión (2015). Esta ley busca modificar parte importante del cuerpo de la LGE y otras leyes asociadas para poner un énfasis en la inclusión y no discriminación en el sistema escolar. Además, busca corregir políticas de selección y exclusión que se daban dentro del sistema escolar público. La atención a las NEE se regula por medio de la Ley Ley $\mathrm{N}^{\circ} 20.248$ sobre Subvención Escolar Preferencial de 2008, y en el caso de los PIE se incluye además el Decreto $\mathrm{N}^{\circ} 170$ del Ministerio de Educación (2009), que regula qué estudiantes pueden recibir dicha subvención. De esta forma, la regulación del sistema de integración tiene una orientación principalmente pecuniaria, es decir, se encarga principalmente de cómo se distribuyen los recursos para atender a los estudiantes que presentan NEE.

El sistema de educación especial atiende a los estudiantes con NEE por medio de dos alternativas. En primer lugar, están las Escuelas Especiales, que son recintos que funcionan de manera paralela al sistema regular y que, por lo general, atienden a estudiantes que requieren de apoyo permanente en áreas específicas derivadas de su condición. Estas escuelas atienden, generalmente de manera exclusiva, a estudiantes con discapacidad visual, auditiva, intelectual, motora, múltiple, trastorno del espectro autista y disfasia severa. Anteriormente, las Escuelas Especiales establecían sus planes y programas en base a decretos específicos para tipos de NEE (Decretos del MINEDUC $\mathrm{N}^{\circ} 86$, 87, 89, 577, 637 y 815). Sin embargo, desde la implementación del Decreto $\mathrm{N}^{\circ} 83$ (2015), a partir del año 2017 las EE deben tomar como referente el currículo nacional con las adecuaciones curriculares que los estudiantes requieran. Los programas de estudio específicos de educación especial anteriormente indicados, permanecen vigentes sólo para la educación media y el ciclo de formación laboral.

La otra modalidad que existe para atender a estudiantes con NEE son los Programas de Integración Escolar (PIE). Estos programas están diseñados para integrar estudiantes con NEE dentro de recintos educacionales regulares, considerando todos los niveles de enseñanza, incluida la modalidad de educación de adultos. Los programas están diseñados bajo un sistema de subvención, que considera la evaluación de los estudiantes por parte de especialistas, y la asignación de recursos específicos que deben ser utilizados para cubrir los gastos asociados a la atención de los estudiantes identificados con NEE. Es así como la existencia del PIE se encuentra sujeta a la especificidad de los casos existentes en la escuela en un período determinado. Según el Decreto $\mathrm{N}^{\circ} 170$ del Ministerio de Educación de 2009, existen ciertas NEE que pueden ser consideradas dentro del PIE, algunas son de carácter permanente y otras transitorias. Para aquellos alumnos que presenten NEE que no están mencionadas en la normativa referida anteriormente, su incorporación a los programas de 
integración se seguirá rigiendo por el Decreto Supremo $\mathrm{N}^{\circ} 1$ del Ministerio de Educación de 1998, que es menos específico respecto de los tipos de NEE que pueden atender los PIE.

Luego del proceso de evaluación inicial, los estudiantes que forman parte del PIE reciben atención especializada según las necesidades detectadas. La atención se realiza principalmente dentro del aula regular por un profesional de apoyo, que debe ser un educador diferencial o especialista validado por el MINEDUC. También se considera el trabajo en el "aula de recursos", que es un espacio diseñado para la atención individual de los estudiantes con NEE. Sin embargo, desde 1998 la prioridad es la atención en el aula regular, Aunque existe que coincide con el llamado de Salamanca a garantizar el acceso al aula regular, lo que responde a la propuesta de Salamanca, que considera esta opción como el medio más eficaz para combatir actitudes discriminatorias, crear comunidades y sociedades integradoras, otorgar educación para todos y mejorar la eficiencia del sistema educacional.

La evaluación inicial especializada es un requisito para ingresar al sistema de Educación Especial, ya sea en una EE o dentro de PIE. Si los padres observan que su hijo o hija necesita estos apoyos extraordinarios, pueden solicitar al profesor a cargo para que, por medio de un informe pedagógico, derive al alumno a los profesionales especialistas correspondientes para que realicen esta evaluación diagnóstica especializada, en caso de que el establecimiento educativo cuente con el PIE y los profesionales adecuados. En caso de que la escuela no cuente con este programa, la evaluación puede ser solicitada en otra escuela que sí cuente con dicho programa o en una escuela de Educación Especial.

Según una guía elaborada por el Ministerio de Educación de 2012, y acorde con la indicación del Marco de Acción en el sentido de coordinar la enseñanza con los sistemas de salud y asistencia social, la evaluación diagnóstica especializada consiste en una evaluación integral tanto del estudiante como de su contex to familiar, escolar y social. De acuerdo a las necesidades observadas, esta evaluación puede ser de tres tipos: (a) evaluación psicoeducativa enfocada en el proceso de aprendizaje y liderada por un educador especial, (b) evaluación especializada no médica, que considera el uso de instrumentos específicos según la condición de los estudiantes por parte de determinados profesionales, y finalmente (c) evaluación de salud, que considera un examen médico general en algunos casos y uno especializado en otros.

En el caso de que la evaluación diagnóstica confirme la presencia de NEE, el alumno será incorporado al PIE, y en caso de no contar con este programa debiera ser derivado a otra escuela. Sin embargo, la con la nueva Ley de inclusión propone un nuevo desafío al sistema, pues por medio del algoritmo de selección es posible que un alumno con NEE sea aceptado en una escuela sin PIE. La incorporación de los niños con NEE al PIE está condicionada por la cantidad de estudiantes con NEE que tenga la escuela. En cada sala de clases no puede excederse el límite de dos estudiantes con NEE permanentes y cinco con NEE transitorias. El establecimiento deberá elaborar un Plan de Apoyo Individual (PAI), el cual presenta la planificación de la(s) intervención(es) que los distintos profesionales llevarán a cabo para responder a las NEE del estudiante, según los resultados del proceso de evaluación correspondiente (MINEDUC, 2013). En el caso de que el estudiante presente necesidades educativas que requieran de adecuaciones curriculares para la consecución de los objetivos de aprendizaje establecidos, se debe elaborar un Plan de Adecuaciones Curriculares Individualizadas (PACI), el cual contiene las medidas de apoyo curricular y orientaciones para el trabajo pedagógico que llevarán a cabo los docentes, incluyendo 
además un seguimiento de la eficacia de las medidas implementadas (Decreto $\mathrm{N}^{\circ} 83$ Exento, 2015). Dicho plan de adecuaciones es realizado tanto para alumnos que se encuentran en escuelas especiales como para alumnos que forman parte de PIE, y contemplan adecuaciones que van desde la presentación de la información hasta eliminación de contenidos, según sea el caso particular.

Por otra parte, en el caso de los recintos hospitalarios que atiendan niños que se encuentran internados por más de tres meses podrán poner en ejecución un aula hospitalaria para asegurar que estos niños puedan mantener una continuidad en cuanto a sus estudios. Esto se puede hacer en dos modalidades, ya sea bajo la creación de una escuela especial en el recinto hospitalario o el establecimiento de un aula hospitalaria que dependa de un recinto educacional que se encuentre en las cercanías del hospital en cuestión. Se busca que los estudiantes participen de actividades que faciliten su posterior reincorporación al sistema escolar y que mejoren su calidad de vida, haciéndolos partícipes de los procesos educativos estipulados por ley.

\subsection{Modelo español ${ }^{5}$}

La legislación española ha experimentado un enriquecido progresivo en materia de educación inclusiva desde la promulgación del Real Decreto de la Ordenación de la Educación Especial en 1985 hasta la actual Ley Orgánica de la Educación (LOE, 2/2006). Esta ley plantea la escolarización sin exclusiones, respondiendo a los principios de calidad y equidad. Uno de los principios esenciales que regula, es el de la atención a la diversidad del alumnado. Este principio debe estar presente a lo largo de toda la enseñanza, teniendo como objetivo el proporcionar una educación que sea adecuada a las características y necesidades de cada alumno (LOE, 2/2006). Además, la LOE establece que los centros educativos deben disponer de los medios que sean necesarios para que todos los alumnos alcancen máximo desarrollo personal, intelectual, social y emocional. Esto se acoge tanto al llamado de Salamanca a la creación de sistemas y programas que tengan en cuenta y celebren las características, intereses y necesidades propias de cada estudiante. Además, a la indicación del Marco de Acción de generar un sistema educativo integrado y una rehabilitación apoyada como métodos para la integración de personas con NEE.

Por otra parte, la Ley Orgánica 8/2013 para la Mejora de la Calidad Educativa (LOMCE), introduce ciertos cambios a la LOE como la incorporación de las evaluaciones externas a lo largo de todas las etapas educativas, o la inclusión de los alumnos con TDA/H, como parte de los alumnos que presentan necesidades específicas de apoyo educativo, entre otras modificaciones. Es así como, tanto la LOE como la LOMCE en conjunto, se constituyen en el marco normativo que reglamenta y organiza el Sistema Educativo español. En base a lo planteado en dicho marco, se entiende entonces que el modelo educativo español está configurado bajo los parámetros de la inclusión.

En este sentido, las medidas establecidas en la legislación española para dar respuesta a las necesidades de todo el alumnado pueden ser de carácter ordinario o de carácter extraordinario. Siguiendo los lineamientos que establece el Ministerio de Educación, Cultura y Deporte, las Comunidades Autonómicas, que tienen transferidas las

\footnotetext{
${ }^{5}$ La información utilizada para la elaboración de este segmento del presente documento fue extraída de la página de la Agencia Ejecutiva en el Ámbito Educativo, Audiovisual y Cultural [EACEA] de la Comunidad Europea: Eurydice (s.f.). España: Apoyo educativo y orientación. Recuperado el 10 de septiembre del 2017, de

https://webgate.ec.europa.eu/fpfis/mwikis/eurydice/index.php/Espa\%C3\%B1a:Apoyo_educativo_y_orientaci\%C3\%B3n
} 
competencias educativas, desarrollan sus propios Planes de Atención a la Diversidad, los cuales incluyen ambos tipos de medidas mencionados anteriormente. Por su parte, los centros educativos, dado que cuentan con autonomía de organización y funcionamiento, ajustan las pautas establecidas por las Comunidades Autonómicas a las necesidades su alumnado y a las características socioeconómicas de su propio contexto escolar, por medio de la elaboración de su Proyecto Educativo de Centro (PEC). El PEC es un documento de carácter pedagógico que está elaborado por la comunidad educativa, el cual define los rasgos que dan la identidad al centro educativo, establece los objetivos que se esperan cumplir y determina su organigrama general. Así, este documento constituye un marco de principios que facilita asumir la diversidad de alumnado que se escolariza, mediante la elaboración de un proyecto curricular que permita dar una respuesta educativa adecuada a los estudiantes, por parte de los docentes y profesionales que trabajan en el centro. Esto se acoge a la indicación del Marco de Acción respecto a que los programas de estudio deben adaptarse a las necesidades de los niños y no al revés, lo que implica entre otras cosas: opciones curriculares para niños con NEE, apoyos adicionales en aula y profesionales externos, flexibilidad escolar, reasignación de recursos, diversificación de las opciones educativas, facilidad para la ayuda mutua entre estudiantes, respaldo a las dificultades y relación con la comunidad y los apoderados.

El sistema educativo español contempla la implementación de distintas medidas para dar respuesta a las diversas necesidades del alumnado. Por una parte, considera medidas ordinarias, que son todas aquellas referidas a la organización general del centro educativo, tales como la distribución de los grupos de alumnos, medidas que favorecen la accesibilidad física al centro, la coordinación y trabajo conjunto entre los distintos profesionales y colaboradores del centro educativo, entre otras. Estas medidas también incluyen aquellas que van en la línea de la prevención y detección de problemas en el aprendizaje, como por ejemplo la aplicación de estrategias de refuerzo y apoyo, o la adaptación a los diferentes ritmos de aprendizaje, entre otras. Por otra parte, existen medidas de carácter extraordinario, que están pensadas para dar respuesta a las necesidades educativas más específicas del alumnado, complementando a las medidas ordinarias. Están dirigidas a aquellos alumnos que, por diferentes razones, encuentran mayores dificultades que el resto en su aprendizaje, por lo que necesitan de una atención educativa que incorpore recursos específicos. Para que las medidas extraordinarias sean aplicadas, es necesario que el alumno cuente con un diagnóstico de las necesidades educativas específicas por medio de una evaluación psicopedagógica que realizan los Equipos de Orientación Educativa y Psicopedagógica (EOEP). Además, se debe hacer un seguimiento continuado del alumno, para ir adaptando las decisiones tomadas y permitir que dicho alumno pueda tener el mayor acceso posible al currículo y la escolaridad regular. Algunas de estas medidas son: adaptaciones significativas del currículo, programas de diversificación curricular, programas de mejora del aprendizaje y del rendimiento, entre otras.

Los EOEP tienen como principal objetivo mejorar la calidad de la educación, principalmente en relación a los alumnos que presentan NEE, interviniendo en centros de educación infantil y primaria. Es destacable que esta intervención responde al área prioritaria de educación pre-escolar propuesta por el Marco de Acción. El trabajo que realizan estos equipos apunta a toda la comunidad educativa: en los centros educativos asesoran el trabajo de los profesores y colaboran en la creación, desarrollo y evaluación de los proyectos educativos del centro; con los alumnos, definen las necesidades tanto 
globales como individuales, y proponen medidas para que el centro educativo responda a ellas; y con las familias, prestan asesoría sobre las estrategias para hacer frente a las dificultades que puedan presentar los niños a lo largo de su etapa escolar.

Los EOEP pueden ser de tres tipos: (1) EOEP De Atención Temprana, que desarrollan acciones preventivas frente a la aparición de trastornos y el agravamiento de discapacidades, detectando y atendiendo problemas del desarrollo en los primeros años de vida; (2) EOEP Generales, que asesoran y colaboran con los centros educativos para el cumplimiento de los objetivos educativos, especialmente a aquellos relacionados a la atención a la diversidad, a los alumnos con necesidades específicas de apoyo educativo, y en la determinación de NEE de los alumnos; y (3) EOEP Específicos, que son equipos con experiencia en una discapacidad en particular, por lo que colaboran con los EOEP generales, con los orientadores pertenecientes a centros específicos, y con los centros de integración preferente.

Pero, además de todos los servicios y labores mencionados que forman parte del quehacer de los EOEP, otra tarea fundamental que tienen estos equipos es la de realizar una propuesta de escolarización para aquellos alumnos que, una vez realizada la evaluación psicopedagógica, presentan NEE. Estos alumnos pueden ser escolarizados en: (a) centros regulares con apoyos educativos específicos, que atienden alumnos que presentan discapacidad motora, sensorial o trastorno generalizado del desarrollo, (b) centros regulares de escolarización preferente, o (c) centros de Educación Especial. Así, estas tres opciones dan cuenta de que existen alternativas educativas que son cada vez más específicas y continuadas, según la influencia de la discapacidad o del trastorno de la conducta en el proceso de aprendizaje y desarrollo individual del alumno.

En el sistema educativo español, la Educación Especial es entendida como una alternativa educativa para aquellos alumnos con NEE que no se beneficiarían de un centro educativo regular, y contemplan la atención desde el nivel preescolar hasta los programas de Transición a la Vida Adulta. Durante la Educación Básica Obligatoria estos centros consideran la realización de adecuaciones curriculares según las necesidades de los estudiantes, y durante el nivel de Transición a la Vida Adulta el foco está puesto en las competencias vinculadas al desempeño profesional y a la inserción social. Respecto de la evaluación, esta se realiza en función de las características de los estudiantes y de los objetivos planteados. La promoción por su parte se realiza según el criterio de los docentes a cargo y es de carácter individual.

Por otra parte, el sistema educativo español no sólo establece apoyos específicos para los alumnos con NEE que cursan su etapa escolar en un centro de Educación Especial, sino que considera también como un alumnado objetivo a aquel que procede de entornos en situación de desventaja social. Específicamente, se consideran aquellos alumnos con condiciones económicas desfavorables, estudiantes de contextos rurales, los hospitalizados, e incluso alumnos que pertenecen a contextos itinerantes, tales como hijos de trabajadores circenses, o de recolectores de fruta, etc. Considerando este último grupo como ejemplo del tipo de medidas que se especifican en la legislación española para estos grupos en desventaja social, se plantea en dicha legislación que las administraciones educativas autonómicas deben garantizar la escolarización de estos alumnos. Esto debe ser logrado por medio de la creación de unidades de apoyo itinerante, la elaboración de programas de escolarización y apoyo educativo para el alumnado que procede de 
colectivos temporeros, y la dotación de un profesor permanente, a quien se le entregan los recursos didácticos básicos.

\subsection{Modelo finlandés ${ }^{6}$}

El sistema educacional de Finlandia está basado en el concepto de equidad y pone énfasis en la adaptación del sistema a los estudiantes y no viceversa como una base fundante de la educación finlandesa. Este foco en equidad y adaptación del sistema a las particularidades del estudiante permite entender el sistema educativo de Finlandia desde la base de la inclusión como lo refiere la misma nación en los documentos presentados a la European Comission: "El sistema educacional en Finlandia ha estado basado en la filosofía de la inclusión desde hace mucho tiempo. La educación básica es igual para todos. No existe una división por habilidades, sino que los estudiantes son apoyados de forma individual para que puedan completar satisfactoriamente su educación básica".

Así se busca que la mayoría de las adecuaciones para estudiantes con NEE se den dentro del aula regular. En casos específicos en los que el apoyo necesario no pueda ser entregado en un aula regular, se opta por la opción de educación en aulas o establecimientos de educación especial, tratando de reducir el tiempo que los estudiantes pasen en esta modalidad. Con estas nociones como modelo de desarrollo, la educación en Finlandia fue reformada el 2011 para establecer tres niveles de apoyo para todo estudiante que lo requiera: apoyo General, Intensificado o Especial.

La idea de estos apoyos es lograr detectar lo más temprano posible la necesidad de ellos por los estudiantes y otorgar apoyo general a todo estudiante que lo necesite dentro del marco de enseñanza regular diaria, es parte de las planificaciones de enseñanza y del proceso de aprendizaje (Basic Education Act, Art. 16). El primer nivel de apoyo o Apoyo general está disponible para todos los estudiantes y está pensado para otorgar mayor tiempo de exposición y repaso de materias a los estudiantes que estén teniendo problemas con un tema o área específica. Así por lo general implica tiempo extra de guía y apoyo durante las actividades cotidianas en la escuela. Además, puede incluir apoyo de enseñanza de refuerzo. En este nivel también se consideran incorporación de educación para necesidades especiales a en formato part-time. Todos estos esfuerzos se pueden aplicar en grupos de enseñanza, en pequeños grupos o de forma individual si así se requiere.

Cuando se habla de educación para necesidades especiales a nivel part-time esto se aplica en situaciones donde hay problemas de aprendizaje lingüístico y matemático, dificultad de aprendizaje en alguna materia específica, habilidades sociales y de aprendizaje o cuando hay un compromiso en la asistencia escolar del estudiante, en este último caso pasa a ser parte del apoyo intensificado.

Aquellos estudiantes que requieran de apoyo de forma regular o a largo plazo en sus procesos de aprendizaje o en referencia a su asistencia a clases, pasan al siguiente nivel que es el de Apoyo Intensificado. También esto se da si es que el estudiante está necesitando apoyo en múltiples formas o áreas de su aprendizaje. El requerimiento de este apoyo debe ser determinado por un equipo multidisciplinar y tener como objetivo central el bienestar del estudiante, referido a buen aprendizaje, buena salud mental y física y el

\footnotetext{
${ }^{6}$ La información utilizada para la elaboración de este segmento del presente documento fue extraída de la página de la Agencia Ejecutiva en el Ámbito Educativo, Audiovisual y Cultural [EACEA] de la Comunidad Europea: Eurydice (s.f.). Finland: Educational Support and Guidance. Recuperado el 10 de septiembre del 2017 , de

https://webgate.ec.europa.eu/fpfis/mwikis/eurydice/index.php/Finland:Educational_Support_and_Guidance
} 
bienestar social, además de las condiciones necesarias para que esto se dé. En este caso se debe diseñar un plan a largo plazo con la colaboración tanto del estudiante como de su tutor legal, padres o apoderados, debe de tomar el Core Curriculum como su base; diseñarse y llevarse a cabo de manera cualitativa y cuantitativa teniendo en consideración las capacidades y necesidades particulares del estudiante (BEA, Art. 16a). El diseño del plan individual por estudiante es la estrategia que se agrega a las ya establecidas en el apoyo general. Este plan busca adecuarse a las particularidades individuales del estudiante y enfrentar dificultades generales que superan la especificidad de una materia o un curso en particular.

Mientras que el apoyo general es un mecanismo de respuesta temprana disponible para todos los estudiantes y el apoyo intensificado busca evitar que los problemas se agraven o se produzcan problemas futuros, el apoyo especial está pensado para aquellos estudiantes que sus particularidades no les permiten lograr los objetivos de aprendizaje mediante el apoyo general o intensificado. Para poder entregar apoyo especial a los estudiantes se debe recopilar información desde profesores y el grupo de bienestar del establecimiento ${ }^{7}$, con esto el proveedor educacional decide si es necesario apoyo especial. Si tal es el caso, esta decisión debe de revisarse al menos dos veces: después del segundo año de educación y antes de que el estudiante pase al séptimo año. Al decidir entregar apoyo especial a un estudiante se decide también su grupo primario de aprendizaje, recursos de intérprete, servicios de asistencia y otros servicios necesarios para que el estudiante en cuestión pueda completar la educación básica obligatoria y pueda acceder a educación secundaria.

El apoyo especial, además de las medidas que están cubiertas en el apoyo general e intensificado, puede contar con el desarrollo de programas de cursos individualizados y aumento o retraso de la educación obligatoria. Antes de que cualquiera de estas medidas pueda ser puestas en marchas se debe conversar acerca de ellas con los padres o apoderados y con el estudiante para informarles de la decisión y escuchar sus opiniones al respecto. Aunque se realiza la consulta a los actores involucrados la decisión de todas formas recae en la autoridad local, pero los padres tienen el derecho a apelar dicha decisión si esta se tomó sin su consentimiento. Como parte del proceso debe llevarse a cabo una evaluación pedagógica la cual puede también estar apoyada por una opinión médica o psicológica o una evaluación social correspondiente (BEA, Art. 17).

Dentro de los tres niveles de apoyo existen medidas estándar que se encuentran disponibles para todos los estudiantes. Todos los niveles de apoyo implican procesos de diferenciación, guía, asistencia personal, servicio de bienestar para los estudiantes (psicólogo escolar) y orientación estudiantil. Además, como se muestra en el cuadro 1 en todos los niveles de apoyo los estudiantes pueden recibir enseñanza recuperativa y educación particularizada para NEE.

Además de las opciones de apoyo especial dentro del marco de la educación regular, existen en Finlandia seis escuelas especiales primarias manejadas por el Estado. Estas escuelas cubren a estudiantes con necesidades derivadas de discapacidades visuales o auditivas importantes, además de otras limitaciones físicas. Estas escuelas junto con cubrir

\footnotetext{
${ }^{7}$ El equipo o grupo de bienestar del colegio es un grupo que debe existir al menos a nivel municipal (a veces localmente en escuelas específicas) y se preocupa de asegurar que existan servicios que apoyen emocional, física y socialmente el bienestar de los estudiantes dentro de los establecimientos. En parte es trabajo preventivo (que cubre las necesidades de los institutos educacionales), en parte relacionado con servicios disponibles (apoyo o cobertura psicológica, médica y social en el colegio). Esto no está cubierto por la Ley General de Educación sino por la Ley de Bienestar de los Niños (No. 417/2007)
} 
la educación de esta población escolar también funcionan como centros de servicio y desarrollo docente en torno al trato de las NEE particulares que cada una de ellas observa, junto con ofrecer apoyo educacional temporal para estudiantes de escuelas regulares.

Cuadro 1. Niveles de apoyo en la educación finlandesa

\begin{tabular}{lccc}
\hline & GENERAL & INTENSIFICADO & ESPECIAL \\
\hline Enseñanza recuperativa & $\mathrm{X}$ & $\mathrm{X}$ & $\mathrm{X}$ \\
Educación para NEE part-time & $\mathrm{X}$ & $\mathrm{X}$ & $\mathrm{X}$ \\
Plan de aprendizaje individual & & $\mathrm{X}$ & $\mathrm{X}$ \\
Programas de aprendizaje personalizados (IEP) & & & $\mathrm{X}$ \\
Extender o atrasar la educación obligatoria & & & $\mathrm{X}$ \\
\hline
\end{tabular}

Fuente: Elaboración propia.

Dentro de la educación especial los rangos de edad son similares a los de la educación regular. La educación obligatoria en Finlandia comienzo el segundo semestre en el año que los estudiantes cumplen 7 años y se extiende por nueve años en un sistema de estructura unificada que integra los cursos de primer ciclo más los cursos menos del ámbito de secundaria. Normalmente es precedida por un año de educación preescolar que se lleva a cabo en guarderías, y no contempla educación formal en lectura o matemática.

Esta etapa de educación básica y educación secundaria temprana es la única que es obligatoria dentro del sistema finlandés. Luego de esta los estudiantes pueden seguir hacia estudios secundarios o estudios técnicos-vocacionales o en algunos casos optar por un año extra de educación básica. Las normas de apoyo particulares presentadas anteriormente están dirigidas casi en totalidad a los estudios de educación ${ }^{8}$ obligatoria. En general, los estudiantes que con NEE pueden participar de educación vocacional (o técnica) a nivel secundario en instituciones tradicionales que imparten este tipo de educación. En tal situación los estudiantes también tienen un plan educacional individual. En la actualidad de los estudiantes con Apoyo Especial que participan de educación vocacional el 88\% lo hace en instituciones regulares de educación vocacional contra un $12 \%$ que participa de este tipo de educación en instituciones especiales.

Además de estas escuelas existen otras siete escuelas básicas que cubren las necesidades educativas de estudiantes con trastornos sociales severos y desordenes emocionales. La educación de estudiantes que están pasando por un tratamiento hospitalario que les impide asistir a una escuela regular está a cargo de la autoridad educativa local del sector donde se ubica el hospital, debiendo hacerse cargo de que los estudiantes puedan seguir su proceso de aprendizaje.

Finalmente, el sistema finlandés de educación enfrenta el desafío de las necesidades especiales también para estudiantes migrantes. Particularmente, en el sistema puede clasificar a los estudiantes a partir de su lengua: se consideran cinco lenguas maternas como posibles del sistema escolar (sueco, finlandés, romaní, sami y lengua de señas), estas lenguas deben ser cubiertas por el sistema y en general sucede así de manera regional, dependiendo de las características de la población local. Los estudiantes que no tienen una de estas lenguas como lengua materna son considerados estudiantes de lengua extranjera,

${ }^{8}$ En el caso de educación secundaria en grandes ciudades muchas escuelas ofrecen también servicios para NEE, en sectores rurales es menos frecuente. 
y pueden recibir Apoyo Especial para las necesidades particulares de aprender en un contexto lingüístico diferente al propio.

\section{Análisis comparado de los tres sistemas}

Aun cuando los tres sistemas tienen similitudes superficiales y se enmarcan en los postulados de la Declaración de Salamanca, son en realidad profundamente diferentes en su conceptualización y tratamiento de las NEE en la educación regular. Las principales diferencias se relacionan con aspectos definitorios de NEE, es decir, con la forma en que se distinguen y categorizan las particularidades de cada estudiante. Asimismo, los tres sistemas difieren en las adaptaciones que se contemplan en los métodos de enseñanza para acoger las diferencias entre estudiantes, y la flexibilidad del sistema para recibir alumnos con NEE en escuelas ordinarias.

\subsection{Aspectos definitorios}

Una primera diferencia entre los tres sistemas es la definición de NEE (cuadro 2), lo que muestra que hay distintas maneras de reconocer las múltiples características que presentan los estudiantes. En el caso chileno, el Decreto 170 establece de manera explícita que existen estudiantes con barreras para aprender o participar de naturaleza permanente o transitoria, a consecuencia de discapacidad o trastorno previamente diagnosticado. Es llamativo que el sujeto del decreto es la Necesidad Educativa Especial como forma de nombrar la diferencia, no el niño que la porta. Esta ontologización de la categoría gnosológica muestra los resabios del modelo médico para el abordaje de las NEE en nuestro país. El sujeto es portador de una condición que solo puede ser validada por medio de un conocimiento experto. No basta con que el estudiante muestre dificultades de rendimiento o problemas en su adaptación escolar. Hay una mirada normalizadora que parte del supuesto que todo estudiante debe adaptarse a un sistema escolar que tiene unos requerimientos específicos y la única manera de tener el derecho a recibir ayudas especiales, es por medio de un reconocimiento formal de que dicha condición no es "normal”.

En el caso español, el sujeto del decreto es el “Alumnado con NEE”, con lo que se nombra la diferencia como una particularidad y no como una desviación. El caso finlandés va más allá, ya que carece de toda definición legal de NEE, y todo niño que se retrase en alguna materia recibe apoyos especiales hasta que sea necesario. Así, un 51\% de los estudiantes finlandeses reciben a lo largo de su escolarización, algún tipo de educación especial. Y por ser más del 50\%, lo especial deja de serlo (Sahlberg, 2011).

Cuadro 2. Comparaciones por país de la definición de NEE

\begin{tabular}{llll}
\hline CHILE & \multicolumn{1}{c}{ ESPAÑA } & FINLANDIA \\
\hline Barreras para aprender y participar que & El alumnado con NEE es “...aquel & No existe una \\
parte del alumnado experimenta durante & que requiera, por un período de su definición & d \\
toda su etapa escolar o en algún & escolarización o a lo largo de toda & legal de NEE. \\
momento de su vida escolar, como & ella, determinados apoyos y & \\
consecuencia de una discapacidad o & atenciones educativas específicas & \\
trastorno diagnosticado. El diagnóstico & derivadas de discapacidad o & \\
es específico y debe considerar ciertos & trastornos graves de conducta” & \\
rangos para definir la presencia de NEE. & (LOE 2/2006, art. 73). & \\
\hline
\end{tabular}

Fuente: Elaboración propia. 


\subsection{Cobertura para la diferencia}

Respecto a la cobertura de las atenciones a las NEE, es interesante constatar una gradiente de generalidad en la definición, desde el caso chileno más bien restrictivo (define exactamente que categorías gnosológicas son consideradas NEE), hasta uno muy general como el caso finlandés que asocia las NEE a los apoyos especiales que cualquier alumno pueda requerir en su proceso educativo (todo niño que requiera, dados sus resultados, apoyos especiales), pasando por el caso español que, si bien es restrictivo, incluye más categorías diferenciadoras, entre ellas los graves problemas de conducta y el talento académico (figura 1). Con esto, vemos que en los tres países se interpreta el principio de Salamanca en relación al reconocimiento de las particularidades de cada estudiante, de manera más o menos restrictiva.

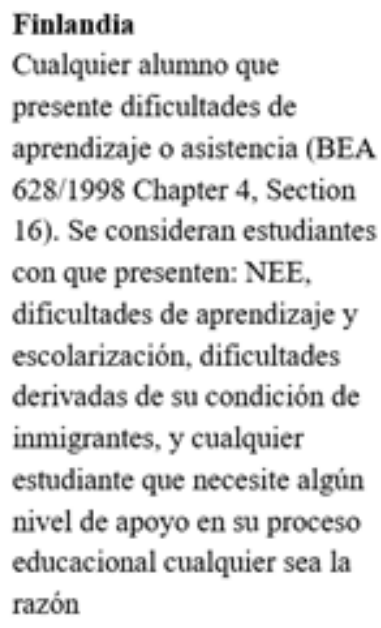

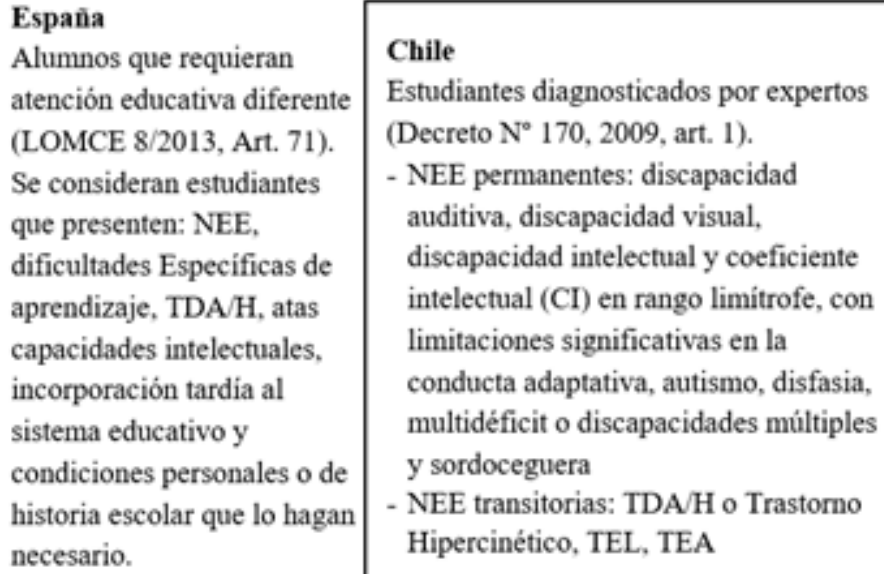

\section{Chile}

Estudiantes diagnosticados por expertos

(Decreto $\mathrm{N}^{\circ} 170,2009$, art. 1).

- NEE permanentes: discapacidad auditiva, discapacidad visual, discapacidad intelectual y coeficiente intelectual (CI) en rango limitrofe, con limitaciones significativas en la conducta adaptativa, autismo, disfasia, multidéficit o discapacidades múltiples y sordoceguera

- NEE transitorias: TDA/H o Trastorno

Hipercinético, TEL, TEA

Figura 1. Comparación de niveles de cobertura

Fuente: Elaboración propia.

En la misma línea del reconocimiento de las características individuales de los estudiantes, vemos que en los tres sistemas revisados el grado de participación de profesores y familias en este proceso obedece a distintos modelos. En un extremo, en el caso chileno, profesores y padres tienen una participación más bien tangencial, pues si bien, son quienes en muchos casos detectan inicialmente la necesidad, la responsabilidad final de la evaluación en todos los casos debe pasar por un especialista (Psicólogos, Fonoaudiólogos, Médicos). Esto implica plantea una visión clínica del diagnóstico y deja de lado la expertise del docente. En el otro extremo, está el caso finlandés, en el cual el diagnóstico de NEE es irrelevante, ya que el profesor es quien toma la decisión de intervenir, basado única y exclusivamente en el rendimiento escolar de los estudiantes. En el caso español, un equipo multidisciplinario, externo a la escuela, es quien hace el diagnóstico y propone los planes de acción remedial. Este equipo se activa ya sea a petición de la escuela, o de las familias que lo requieran.

Respecto a la entrega de una enseñanza adecuada y de calidad según cuales sean las características de los estudiantes, vemos que en los tres países se trabaja en consecuencia con la forma que cada uno tiene de entender las necesidades educativas especiales. En 
Chile, el involucramiento de profesores está normado por los PIE que promueven un modelo de co-docencia, en el cual hay siempre un profesor especialista a cargo de los niños con NEE en el aula integrada. Como se ha indicado antes, esto puede implicar que el profesor esté a cargo de varios estudiantes, con NEE específicas muy diferentes. En la práctica, lo que esto implica, es que el aula PIE es un aula aparentemente inclusiva, pero con una segregación real, en dos cursos con dos profesores, que habitan una misma sala. En el otro extremo, el caso Finlandés trabaja intensivamente con los niños integrados con NEE en el aula de recursos, y los hace participar del aula regular solo en las actividades en las que los profesores consideran que pueden obtener un verdadero beneficio académico o social. El caso español es un caso intermedio, en el que el equipo asesor externo se encarga de las adecuaciones materiales para que el estudiante se vea beneficiado del aula regular. Y si ello no es posible, el estudiante es atendido en un sistema segregado.

Cuadro 3. Grado de involucramiento de los diferentes actores responsables de la detección e intervención de NEE por país

\begin{tabular}{llll}
\hline & \multicolumn{1}{c}{ CHILE } & \multicolumn{1}{c}{ ESPAÑA } & FINLANDIA \\
\hline $\begin{array}{l}\text { Encargado del } \\
\text { diagnóstico de la NEE }\end{array}$ & $\begin{array}{l}\text { A cargo de un especialista } \\
\text { específico, de acuerdo a la } \\
\text { normativa vigente }\end{array}$ & $\begin{array}{l}\text { Equipo } \\
\text { multidisciplinario }\end{array}$ & Profesor* \\
\hline $\begin{array}{l}\text { Encargado del } \\
\text { tratamiento o } \\
\text { intervención de NEE }\end{array}$ & $\begin{array}{l}\text { Especialista + profesor de } \\
\text { educación diferencial }+ \\
\text { profesor de aula regular }(\mathrm{en} \\
\text { menos medida). }\end{array}$ & $\begin{array}{l}\text { Equipo } \\
\text { multidisciplinario }+ \\
\text { profesor }\end{array}$ & $\begin{array}{l}\text { Profesor }+ \\
\text { equipo } \\
\text { de apoyo }\end{array}$ \\
\hline
\end{tabular}

Nota: (*) En caso de que el profesor lo requiera, el diagnóstico pedagógico puede acompañarse de un diagnóstico psicológico o médico, pero este no es obligatorio para implementar las medidas de apoyo.

Fuente: Elaboración propia.

\subsection{Flexibilidad del sistema para recibir a niños con $\mathrm{NEE}$ en las escuelas regulares}

Finalmente, vemos que en los tres países hay grandes diferencias respecto al acceso que tienen los niños con NEE a las escuelas regulares. Aun cuando la Declaración de Salamanca es enfática en señalar que se debe asegurar la aceptación de todos los niños en aulas ordinarias, vemos que a diferencia de los casos europeos estudiados, en Chile es el niño el que debe adaptarse al sistema: si el colegio al que postula no tiene un programa PIE específico a su condición, debe buscar otro colegio. Si bien, con la nueva ley de inclusión, es posible que un niño sea aceptado en un colegio sin programa PIE, el niño no recibirá en él los apoyos oportunos y pertinentes que hagan posible una plena inclusión educativa como lo establece la Declaración de Salamanca. Y si el colegio es privado, el establecimiento no está obligado a recibir al niño (cuadro 4).

Cuadro 4. Flexibilidad del sistema escolar para recibir a estudiantes con NEE

\begin{tabular}{lll}
\hline \multicolumn{1}{c}{ CHILE } & \multicolumn{1}{c}{ ESPAÑA } & \multicolumn{1}{c}{ FINLANDIA } \\
\hline En teoría, muy flexible, ya & Flexible con obligatoriedad & Flexible con planes de apoyo \\
que la Ley de Inclusión & de adaptación curricular & particulares para cada \\
obliga a las escuelas a recibir & particularizada. & estudiante. \\
a todos los niños. En la & Adaptaciones desde el & Adaptación del currículum a \\
práctica, inflexible, ya que los & sistema a las necesidades del & las necesidades particulares \\
colegios solo atienden & estudiante. & del estudiante. \\
adecuadamente a los niños & Aulas abiertas de EE en & \\
para los cuales tienen PIE. & centros ordinarios. & \\
\hline
\end{tabular}

Fuente: Elaboración propia. 


\section{Conclusiones: ¿De qué inclusión hablamos en Chile cuando hablamos de inclusión?}

De la sucinta y sinóptica comparación entre los tres sistemas que acabamos de exponer, vamos a concluir desde el caso chileno, que es claramente el que muestra las principales falencias tanto conceptuales, como reglamentarias y reales, en relación a la declaración de Salamanca.

En primer lugar, parece claro que, en los países más desarrollados, existe el supuesto básico que es obligación de toda escuela recibir a los niños que demanden atención educativa, sin discriminación de ningún tipo por condición de NEE, y sin ninguna barrera burocrática adicional que impida que esto ocurra realmente. En Chile, lamentablemente, todo niño con NEE debe pasar al menos por tres barreras burocráticas para recibir atención educativa: a) que la escuela cuente con PIE en caso de ser municipal, o con equipos profesionales de apoyo, en caso de ser particular, b) que el niño tenga un diagnóstico especializado que le permita ser portador de beneficio PIE, y c) que en la escuela a la que quiere ingresar exista un PIE adecuado a la particular condición del niño.

En segundo lugar, es importante destacar que, al operar de esta manera, el sistema chileno tiene una estructura burocrática y reglamentaria que hace que las normativas pasen a ser más importantes que el niño que está siendo incorporado al sistema. Sin diagnóstico no hay PIE. Y sin PIE no hay recursos para contratar especialistas. Y sin especialistas no hay recursos para atender al niño. En otras palabras, el niño que debe ser educado, es sujeto de educación condicional mientras no se cumplan con los requisitos administrativoburocráticos que le den un sello de educabilidad. Las familias que tienen niños con NEE pasan por este proceso en múltiples oportunidades durante el proceso de educación formal, y ante cualquier cuestionamiento, las autoridades aluden a que esa es la configuración del sistema, y que lo por lo tanto, las familias deben adaptarse a ello.

En el ámbito de las Escuelas Especiales, su realidad no es tan diferente. El principio de inclusión obliga a estas escuelas a compartir el currículum nacional de enseñanza básica, con las debidas adecuaciones curriculares. O presentar una propuesta de currículum alternativo, que puede llevar años en ser aprobado, lo que lleva a que muchas EE deban cerrar si fallan en el intento. Por ello, gran cantidad de las EE optan por el camino de las adecuaciones curriculares. Pero este camino obliga, sorprendentemente, a que los niños de todas las escuelas especiales, tengan que seguir todas las materias del currículum de enseñanza básica. Esto muchas veces genera un distanciamiento de los objetivos curriculares respecto de las necesidades de los estudiantes, lo que se puede ver por ejemplo, en el caso de una escuela de retos múltiples que atiende a estudiantes sordociegos, donde se deben definir actividades para la enseñanza del inglés.

¿Y qué muestran de fondo estas prácticas del caso chileno? Creemos que una visión restrictiva del concepto de inclusión. Una visión que muestra la mala comprensión de este concepto. Una comprensión parcial. En la que se piensa que la inclusión significa que todos deben estar siempre juntos y que todos pueden y deben hacer lo mismo. Lo que paradojalmente, lleva a una educación normalizadora y uniformizadora. Una educación en la que algunos son educados, y otros son discriminados de la peor forma posible: son invisibilizados en sus verdaderas necesidades educativas, porque el sistema juega a que están plenamente incluidos. 


\section{Referencias}

Agencia Ejecutiva en el ámbito Educativo, Audiovisual y Cultural. (2015). España: Apoyo educativo y orientación. Recuperado de https://webgate.ec.europa.eu/fpfis/mwikis/eurydice/index.php/Espa\%C3\%B1a:Apoy o_educativo_y_orientaci\%C3\%B3n

Agencia Ejecutiva en el ámbito Educativo, Audiovisual y Cultural. (2015). Finland: Educational support and guidance. Recuperado de https://webgate.ec.europa.eu/fpfis/mwikis/eurydice/index.php/Finland:Educational_ Support_and_Guidance

MINEDUC. (2013). Orientaciones técnicas para programas de orientación escolar (PIE). Recuperado de https://especial.mineduc.cl/wp-content/uploads/sites/31/2016/09/Orientaciones- PIE2013-3.pdf

Rosas, R., Staig, J. y Lazcano, G. (2018). Invisibilizar la diferencia: El desafío que nos falta para tener una educación verdaderamente inclusiva en Chile. En I. Sánchez (Ed.), Ideas en Educación II. Definiciones en tiempos de cambio (pp. 685-708). Santiago de Chile: Ediciones UC.

Sahlberg, P. (2011). Finnish lessons. Nueva York, NY: Teachers College Press.

UNESCO. (1994). Declaración de Salamanca y marco de acción para las necesidades educativas especiales. París: UNESCO.

\section{Breve CV de los autores}

\section{Ricardo Rosas}

Psicólogo de la Pontificia Universidad Católica de Chile y Doctor en Psicología Cognitiva de la Freie Universität Berlin. Profesor Titular de la Escuela de Psicología de la PUC. Sus áreas de interés son la Inteligencia, la Psicología de las Discapacidades, el Juego y Aprendizaje Implícito y el desarrollo de sistemas instruccionales basados en tecnologías. Es autor de cuatro libros y numerosas publicaciones en revistas internacionales en sus temas de investigación. Actualmente dirige el Centro de Desarrollo de Tecnologías de Inclusión de la PUC, CEDETi-UC y la línea de Inclusión y Discapacidad del Centro de Justicia Educacional (CJE) de la PUC. ORCID ID: https://orcid.org/oooo-0002-30914044. Email: rrosas@uc.cl

\section{James Staig}

Investigador independiente $\mathrm{y}$ Doctor en Literatura y Estudios Culturales Latinoamericanos de The University of Texas at Austin. Ha desarrollado investigación en áreas de Literatura y sonido, soportes y metodologías de inclusión en el estudio literario, discapacidad y literatura y discapacidad e inclusión en contextos educacionales. Es fundador de la editorial nacional de audiolibros Leolento con la cual ha desarrollado proyectos de inclusión para personas con discapacidad visual en contexto nacional. Actualmente realiza labores de docencia en la Universidad Adolfo Ibáñez. ORCID ID: https://orcid.org/OOOO-O001-8947-0719.Email: jstaig@gmail.com 


\section{Guillermo Lazcano}

Psicólogo de la Pontificia Universidad Católica de Chile y Máster en Neurociencia de la Universidad Complutense de Madrid. Editor Ejecutivo de la revista Estudios de Psicología de la Fundación Infancia y Aprendizaje, y colabora en investigaciones con CEDETi-UC y el Centro de Desarrollo de Tecnología Biomédica (CTB) de la Universidad Politécnica de Madrid. Sus áreas de interés e investigación comenzaron con las políticas educativas, aunque actualmente se ha enfocado en procesos de adicción y el efecto de las drogas en el cerebro. ORCID ID: https://orcid.org/0000-0003-1694-261X.

\section{Rosario Palacios}

Socióloga de la Pontificia Universidad Católica de Chile, Magíster en Planificación Urbana de Columbia University y Doctora en Sociología de London School of Economics. Investigadora asociada del Centro de Justicia Educacional de la PUC y dicta la cátedra de Políticas Públicas en la carrera de Antropología de la misma universidad. Sus áreas de interés son las políticas públicas, la metodología etnográfica para su estudio, diseño y evaluación, y las implicaciones espaciales en su implementación. Su trabajo ha sido publicado en revistas académicas nacionales e internacionales. ORCID ID:

https://orcid.org/0000-0001-8839-3520.Email: r.palacios@uc.cl

\section{Victoria Espinoza}

Profesora de Educación Básica, especialista en Dificultades de Aprendizaje, Magíster en Psicología de la Pontificia Universidad Católica de Chile. Actualmente es candidata a Doctora en Psicología de la misma universidad. Sus áreas de interés con el desarrollo de la lectura, las funciones ejecutivas, la educación inclusiva y las brechas de aprendizaje según el nivel socioeconómico. Actualmente se desempeña como encargada del área de investigación del Centro de Desarrollo de Tecnologías de Inclusión y participa como estudiante de doctorado en el Centro de Justicia Educacional. ORCID ID: https://orcid.org/0000-0001-9462-1139. Email: victoriaespinoza@uc.cl

\section{Mikko Aro}

Neuropsicólogo de la Vrije Universiteit de Amsterdam, Holanda, Doctor en Psicología de la Universidad de Jyväskylä, Finlandia. Es professor titular del Departamento de Educación e la Universidad de Jyväskylä. Sus áreas de interes son la enseñanza y el aprendizaje, el desarrollo cognitivo, el desarrollo del lenguaje y la educación inclusiva entre otras. Ha desarrollado un importante trabajo respecto de la investigación de la fluidez, tanto de la lectura como de las matemáticas. También ha investigado el rol de la autoeficiacia en el processo lector. En su labor docente se há enfocado en el campo de la educación especial, realizando tanto cursos como supervisión de tesis de magister y de doctorado. ORCID ID: https://orcid.org/0000-0002-0545-0591. Email:

mikko.t.aro@jyu.fi

\section{Candelaria Imbernón}

Es licenciada en Filosofía y Ciencias de la Educación y Doctora en Pedagogía de la Universidad de Murcia. Es especialista en sistemas alternativos de comunicación y tecnologías de apoyo para personas con discapacidad. Es profesora titular del Centro Universitario La Salle en España. Sus intereses de investigación se orientan al diseño de 
aplicaciones tecnológicas para la enseñanza de la lectura y la escritura de niños con necesidades de apoyo educativo. Imparte docencia en el área de evaluación e intervención de niños con discapacidad motora y sensorial, y realiza labores en otros centros universitarios de España, tales como la Universidad Complutense de Madrid. ORCID ID: https://orcid.org/0000-0001-7882-0592. Email: c.imbernon@lasallecampus.es 